\title{
Stellar velocity dispersions and emission line properties of SDSS-III/BOSS galaxies
}

\author{
D. Thomas ${ }^{1,2}$, O. Steele ${ }^{1}$, C. Maraston ${ }^{1,2}$, J. Johansson ${ }^{1,3}$, \\ A. Beifiori ${ }^{1,4}$, J. Pforr ${ }^{1,5}$, G. Strömbäck ${ }^{1}$, C. A. Tremonti ${ }^{6}$, D. Wake ${ }^{7}$ \\ and the BOSS collaboration \\ ${ }^{1}$ ICG, University of Portsmouth, Dennis Sciama Bldg, Burnaby Road, PO1 3FX, UK \\ ${ }^{2}$ SEPnet, South East Physics Network, (www.sepnet.ac.uk) \\ ${ }^{3}$ Max-Planck-Institut für Astrophysik, D-85748 Garching, Germany \\ ${ }^{4}$ MPI für extraterrestrische Physik, Giessenbachstraße, D-85748 Garching, Germany \\ ${ }^{5}$ NOAO, 950 North Cherry Ave., Tucson, AZ 85719, USA \\ ${ }^{6}$ Department of Astronomy, University of Wisconsin-Madison, Madison, WI 53706, USA \\ ${ }^{7}$ Yale Center for Astronomy and Astrophysics, Yale University, New Haven, CT, USA
}

\begin{abstract}
We perform a spectroscopic analysis of 492,450 galaxy spectra from the first two years of observations of the Sloan Digital Sky Survey-III/Baryonic Oscillation Spectroscopic Survey (BOSS) collaboration. This data set has been released in the ninth SDSS data release, the first public data release of BOSS spectra. We show that the typical signal-to-noise ratio of BOSS spectra is sufficient to measure stellar velocity dispersion and emission line fluxes for individual objects. The typical velocity dispersion of a BOSS galaxy is $240 \mathrm{~km} / \mathrm{s}$, with an accuracy of better than 30 per cent for 93 per cent of BOSS galaxies. The distribution in velocity dispersion is redshift independent between redshifts 0.15 and 0.7 , which reflects the survey design targeting massive galaxies with an approximately uniform mass distribution in this redshift interval. The majority of BOSS galaxies lack detectable emission lines. We analyse the emission line properties and present diagnostic diagrams using the emission lines [OII], $\mathrm{H}_{\beta}$, [OIII], Halpha, and [NII] (detected in about 4 per cent of the galaxies). We show that the emission line properties are strongly redshift dependent and that there is a clear correlation between observed frame colours and emission line properties. Within in the low- $z$ sample around0.15 $<z<0.3$, half of the emission-line galaxies have LINER-like emission line ratios, followed by Seyfert-AGN dominated spectra, and only a small fraction of a few per cent are purely star forming galaxies. AGN and LINER-like objects, instead, are less prevalent in the high- $z$ sample around $0.4<z<0.7$, where more than half of the emission line objects are star forming. This is a pure selection effect caused by the non-detection of weak $\mathrm{H}_{\beta}$ emission lines in the BOSS spectra. Finally, we show that star forming, AGN and emission line free galaxies are well separated in the $g-r$ vs $r-i$ target selection diagram.
\end{abstract}

Keywords. surveys, galaxies: general, galaxies: evolution, galaxies: kinematics and dynamics, galaxies: ISM, galaxies: active evolution

\section{Introduction}

Recent large galaxy surveys have opened a new avenue in observational galaxy evolution, by providing large data sets that allow statistical studies of large galaxy samples in the nearby universe. In the last decade an overwhelming number of studies based on data from the Sloan Digital Sky Survey (York et al. 2000) have led to significant progress in our understanding of the local galaxy population. The Baryon Oscillation Spectroscopic Survey (hereafter BOSS, cite[Dawson et al. 2012]Dawson12), part of the Sloan Digital Sky Survey III collaboration Eisenstein et al. (2011), is extending this database to higher redshifts obtaining spectra of 1.5 million luminous galaxies up to redshifts $z \sim 0.7$ by 

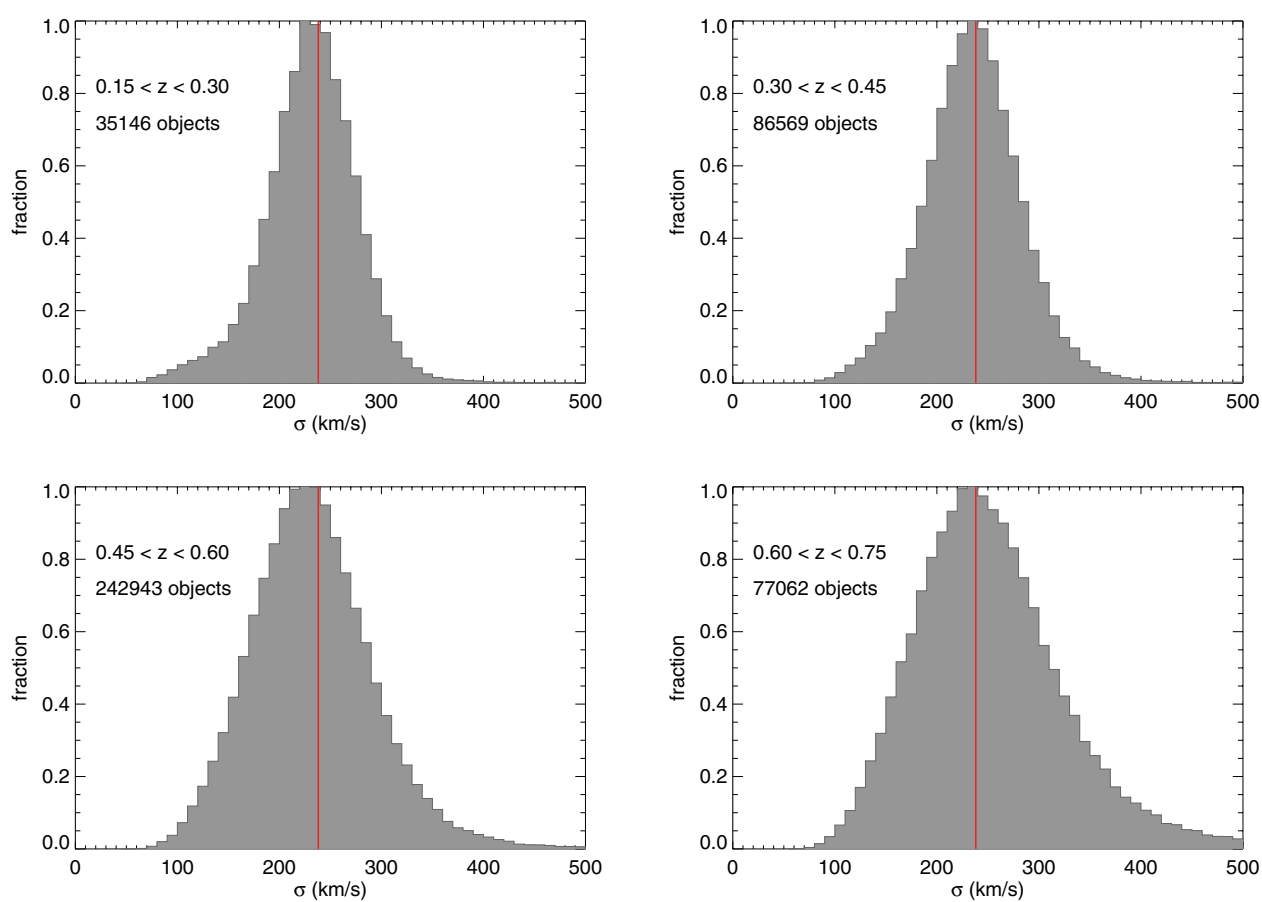

Figure 1. Distribution in velocity dispersion for spectra with an error of less than 30 per cent in the redshift intervals $0.15<z \leqslant 0.3,0.3<z \leqslant 0.45,0.45<z \leqslant 0.6,0.6<z \leqslant 0.75$. The red line indicates the position of the peak for the four central redshift intervals. The distribution is approximately Gaussian and there is no evolution within $0.15<z<0.75$.

2014. This will allow large-scale statistical studies of the galaxy population at an epoch when the universe had only half of its current age. The present work is published in Thomas et al. (2013).

\section{Data and analysis tools}

We use the data from the first two years of BOSS observations published in the first BOSS data release DR9; Ahn et al. 2012 containing 534,474 galaxy spectra from 813 plates over $3,275 \mathrm{deg}^{2}$ on the sky. We select those objects that are classified as galaxies and for which the ZWARNING_NQSO flag is set zero (reliable redshifts and excluding QSOs) resulting in 492,450 unique objects (excluding repeat observations). This includes all BOSS galaxies from DR9 that have been selected through the LOWZ and CMASS selection cuts. The SPARSE sample is included within the CMASS selection. We do not consider LOWZ or CMASS targets with spectra from SDSS I/II.

We use the publicly available codes pPXF Cappellari \& Emsellem (2004) and GANDALF v1.5 Sarzi et al. (2006) to calculate stellar kinematics and to derive emission line properties. The stellar population templates are adopted from Maraston \& Strömbäck (2011). For more detail about the data sample and the analysis tools we refer the reader to Thomas et al. (2013).

\section{Velocity dispersions of BOSS galaxies}

In the following we present the measurements of the line-of-sight velocity dispersions and discuss their redshift evolution. Fig. 1 shows the distributions of velocity dispersions 

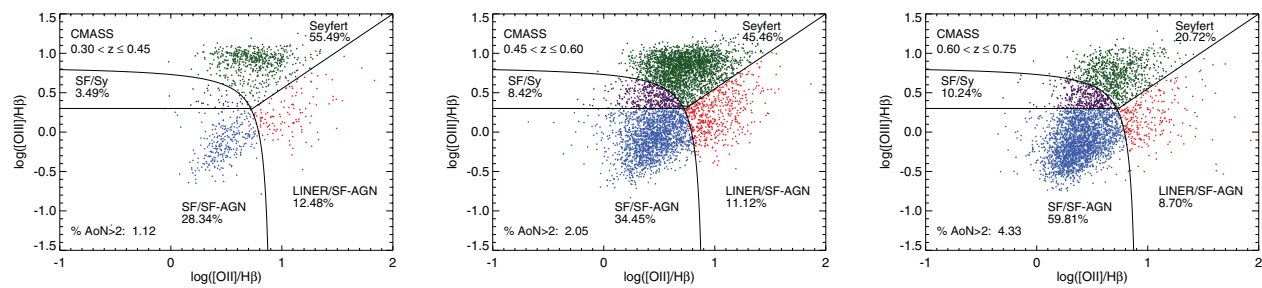

Figure 2. Emission line classification (Lamareille 2010) for CMASS BOSS galaxies. The fraction of objects for which all three emission lines are detected (requiring the amplitude-over-noise ratio of all three lines to be larger than two) is given in the bottom-left corner of each panel. The fractions of galaxies in each of the emission line classes are given by the labels in the panels. The relative fractions of star forming and AGN in the redshift interval $0.3 \leqslant z \leqslant 0.45$ agree well with the results form the BPT classification. Beyond those redshifts, the fraction of star forming CMASS galaxies increases considerably with increasing redshift.

in the redshift intervals $0.15-0.3,0.3-0.45,0.45-0.6$, and $0.6-0.75$. The red line indicates the mean position of the peak. The distribution in velocity dispersion is largely independent of redshift within $0.15<z<0.75$. The position of the peak of the distribution does not change, while the width becomes somewhat larger at higher redshifts caused by slightly larger measurement errors see also Shu et al. 2011. This uniformity of the distribution in velocity dispersion as a function of redshift between 0.15 and 0.75 is an important feature of the BOSS target selection, as it establishes a direct link between the galaxy samples at various redshifts, which allows us to accurately probe the redshift evolution of massive galaxies. Target selection in BOSS has been designed to this purpose and, using stellar velocity as proxy for galaxy mass, the present results shows that this goal has been achieved.

\section{Emission line properties of BOSS galaxies}

In this section we present the emission line properties of our BOSS galaxy sample. The aim is to discuss the prevalence of various galaxy types and emission line classes in the BOSS target selection algorithm. We use the emission lines $\mathrm{H}_{\beta}$, [OIII $] \lambda 5007, \mathrm{H}_{\alpha}$, and $[\mathrm{NII}] \lambda 6583$ to perform the standard classification based on the BPT diagnostic diagram first introduced by Baldwin et al. (1981) for galaxies with redshifts $z<0.45$ (140,596 objects). Galaxies at higher redshifts, instead, are analysed using the blue emission line diagnostics by Lamareille (2010), which seems to be most comparable to the approach used for redshifts $z<0.45$. In both cases we only include objects where the full set of key diagnostic emission lines can be detected with an amplitude over noise ratio above 2. This is the case for about 3 per cent of the BOSS galaxy population.

Fig. 2 presents the results for the redshift intervals $0.3<z<0.45,0.45<z<0.6$, and $0.6<z<0.75$, equivalent to the BPT diagnostic at lower redshifts. The fractions of galaxies in each of the emission line classes are given by the labels in the panels. The relative fractions of star forming/SF-AGN composite and AGN in the redshift interval $0.3 \leqslant z \leqslant 0.45$ agree well with the results from the BPT classification. Beyond those redshifts, the fraction of star forming CMASS galaxies increases considerably with increasing redshift. At redshifts above 0.6, almost two thirds of the CMASS galaxies with detected emission lines are star forming or SF-AGN composites. This trends is most probably due to the fact that the $\mathrm{H}_{\beta}$ emission line is the weakest among those three, so that objects with low $\mathrm{H}_{\beta}$ fluxes drop out first with increasing redshift. These are AGN, while star forming galaxies with stronger $\mathrm{H}_{\beta}$ emission stay above the AoN threshold. A scientific analysis aimed at studying relative fractions of star forming and AGN with redshift will 
need to perform a careful assessment of this selection effect, which goes beyond the scope of this paper. Still, the BOSS sample is certainly useful for the identification and selection of massive star forming galaxies at redshifts around $z \sim 0.6$.

\section{Correlations between observed frame colours and emission line properties}

For the CMASS sample at redshifts above $z \sim 0.4$, the separation of the various emission-line classes is well pronounced in the $g-r$ vs. $r-i$ colour space. There are clear correlations between the observed frame colours and the emission-line properties of CMASS galaxies. There is a strong bias such that CMASS galaxies without emission lines are predominantly found in the classical LRG section at red $g-r$ colours. CMASS galaxies with emission lines, instead, are more likely to be found at blue $g-r$ colour, and galaxies with star formation activity, in particular, populate the blue end of the $g-r$ colour space, typically having $(g-r)<1.0$. AGN, both Seyfert and LINER, preferentially occupy intermediate $g-r$ colour in between those two extremes.

The dependence of star formation activity on observed $g-r$ colour for the CMASS galaxies as found in the present work is not surprising. It was to be expected that the blue $g-r$ colour is driven by the presence of young stellar populations caused by recent or current star formation episodes. Tojeiro et al. (2012) analyse the star formation histories of CMASS galaxies through spectral SED fitting and find extended star formation in blue CMASS galaxies. The present results confirms this finding. If the CMASS galaxies with blue $g-r$ are dominated by young stellar populations and have a fraction of star forming galaxies, one would expect to find mostly late-type galaxies. Indeed, Masters et al. (2011) analyse the morphologies of BOSS galaxies through HST/COSMOS imaging and find that most CMASS galaxies with blue $g-r$ colour are in fact late type systems.

To conclude, BOSS offers spectra of a large sample of galaxies up to redshifts $\sim 0.8$. The quality of BOSS spectroscopy, even though designed for redshift determination, allows the measurement of simple quantities on individual BOSS spectra for a wealth of galaxy evolution studies on dynamical, gas, and stellar population properties.

\section{References}

Ahn C. P., et al., 2012, ApJS, 203, 21

Baldwin J. A., Phillips M. M., \& Terlevich R., 1981, PASP, 93, 5

Cappellari M., Emsellem E., 2004, PASP, 116, 138

Dawson K. S., et al., 2013, AJ, 145, 10

Eisenstein D. J. et al., 2011, AJ, 142, 72

Lamareille F., 2010, A\&BA, 509, 53

Maraston C., Strömbäck G., 2011, MNRAS, 418, 2785

Masters K., et al., 2011, MNRAS, 418, 1055

Sarzi M. et al., 2006, MNRAS, 366, 1151

Shu Y., et al., 2011, ApJ, 143, 90

Thomas D. et al., 2013, MNRAS, 431, 1383

Tojeiro R., et al., 2012, MNRAS, 424, 136

York D. G., et al., 2000, AJ, 120, 1579 